\title{
Caracterización de antígenos de bajo peso molecular de Angiostrongylus costaricensis, reconocidos durante una infección experimental en roedores
}

\author{
ELIZABETH S. ABRAHAM ${ }^{1,2}$, HARTWIG SCHULZ-KEY ${ }^{2}$ y STEFAN M. GEIGER ${ }^{2,3}$
}

\section{CHARACTERIZATION OF LOW MOLECULAR WEIGHT ANTIGENS OF Angiostrongylus costaricensis RECOGNIZED DURING AN EXPERIMENTAL INFECTION IN MICE}

The immune response to low molecular weight components of Angiostrongylus costaricensis somatic crude antigen was investigated. During the chronic phase of infection three immunodominant antigens: 20, 15 and $2 \mathrm{kDa}$, were recognized by IgG and IgG1 using the Western blot method. In order to obtain the initial amino acid sequences of each recognized antigen, the Edmann digestion method was utilized. The sequence of $15 \mathrm{kDa}$ antigen was found to have 100\% homology with the Glutation-S-transferase enzyme of Brugia malayi and Onchocerca volvulus, and the sequence of $2 \mathrm{kDa}$ antigen $95 \%$ homology with the Ubiquitine protein. For the $20 \mathrm{kDa}$ antigen, a sequence of thirteen amino acids was obtained and it did not show homology to any protein described up to date. The cross reactivity to these components was weak, particularly to the $20 \mathrm{kDa}$ component, to which no cross reactivity is detected. We propose further studies with human sera in order to investigate the usefulness of these antigen in the development of a specific diagnostic test for abdominal angiostrongylosis.

Key words: Angiostrongylus costaricensis, Antigens, Immunology.

\section{INTRODUCCIÓN}

Angiostrongylus costaricensis (Morera y Céspedes 1971) es el agente etiológico de la angiostrongilosis abdominal, una zoonosis que se encuentra distribuida en Latinoamérica, desde México hasta el sur de Brasil. Los hospederos definitivos naturales del parásito son roedores de las especies Sigmodon hispidus, Rattus rattus y Oryzomis spp; los hospederos intermediarios son moluscos de la familia Veronicellidae. El habitat final del parásito son las arterias mesentéricas, principalmente a nivel de la región ileocecal.

La infección en el ser humano se caracteriza por una fuerte reacción inflamatoria a nivel de la pared intestinal y por la aparición de una serie de síntomas inespecíficos tales como: vómito, anorexia, dolor en el cuadrante inferior derecho, estreñimiento y ocasionalmente fiebre ${ }^{1,2}$. Los casos severos pueden conducir a la muerte, sobre todo si el cuadro se presenta en niños ${ }^{3,4}$. Debido a la fuerte reacción inflamatoria las formas larvales

\footnotetext{
Department of Parasitology, University of Costa Rica, San Pedro/San José, Costa Rica.

Institute of Tropical Medicine, University of Tübingen, Wilhelmstr. 27, 72074 Tübingen, Germany.

3 Institute for Comparative Tropical Medicine and Parasitology, University of Munich, Leopoldstr. 5, D-80802 Munich Germany.
} 
de primer estadio (L1) no son capaces de alcanzar la luz intestinal, por lo que un diagnóstico coproparasitológico de la infección es imposible. Hasta la fecha, sólo se puede confirmar la presencia del parásito en preparados histológicos enviados al laboratorio, luego de una intervención quirúrgica. Como métodos diag-nósticos complementarios se emplean en el laboratorio clínico pruebas inmunológicas que intentan determinar la presencia de anticuerpos específicos contra A. costaricensis en el suero de los pacientes sospechosos ${ }^{1,5,6}$. Estas pruebas presentan sin embargo, el gran inconveniente de las reacciones cruzadas, las cuales afectan la especificidad y por ende la confiabilidad de las mismas. Nuestros trabajos experimentales señalan que gran parte de esta reacción cruzada se produce contra antígenos de alto peso molecular, entre los $80 \mathrm{y}$ $220 \mathrm{kDa}$ (datos no publicados), observándose una mayor especificidad para los componentes de bajo peso molecular, especialmente bajo los $50 \mathrm{kDa}$. En el presente estudio se procede al análisis y caracterización de la respuesta inmune humoral contra la fracción de antígeno somático entre los $30 \mathrm{kDa}$ y $1,0 \mathrm{kDa}$, durante una infección experimental con A. costaricensis.

\section{MATERIAL Y MÉTODOS}

Se utilizaron ratones de la cepa BALB/c $(\mathrm{n}=$ 17) y C57BL/6 $(n=11)$ con edades entre los 2 y 4 meses. Los animales fueron suministrados por el laboratorio Charles River, Sulzfeld-Alemania y mantenidos en el Instituto de Medicina Tropical (Tuebingen-Alemania) bajo las condiciones estándares de laboratorio.

Larvas de tercer estadio (L3) de $A$. costaricensis se obtuvieron y aislaron a partir de moluscos de la especie Biomphalaria glabrata ${ }^{7}$. Cada ratón fue inoculado en forma oral con seis L3. A partir de formas adultas de A. costaricensis, recuperadas de arterias mesentéricas de animales infectados, se prepara un extracto de antígeno somático de la siguiente manera: 50 a 60 machos y hembras del parásito fueron macerados manualmente con $0,1 \mathrm{ml}$ de $\mathrm{PBS}$ en un macerador de tejido (GLT, Kirchheim/Teck, Alemania). El macerado fue recolectado en tubos Eppendorf de $1,5 \mathrm{ml}$ y se agregó PBS hasta completar $1 \mathrm{ml}$. El material fue sometido a tres ciclos de sonicación con un intervalo de dos minutos entre cada ciclo (Branson Sonifier 250, Danburry,
Conn). Luego se centrifugó la suspensión a $16.000 \mathrm{rpm}, \mathrm{a} 4{ }^{\circ} \mathrm{C}$, por 20 minutos. Se recolectó el sobrenadante y se determinó la concentración de proteína ${ }^{8}$, utilizando albúmina bovina como solución estándar. Se prepararon alícuotas de 0,5 $\mathrm{ml}$ del antígeno que se almacenaron a $-20^{\circ} \mathrm{C}$.

Los sueros de los animales infectados con $A$. costaricensis fueron obtenidos por punción del plexo venoso retroorbital, según procedimiento descrito previamente ${ }^{9}$. Estos sueros fueron obtenidas semanalmente de cada animal y para el ensayo se usaron sólo los de los animales que sobreviven hasta el final del experimento.

Para medir la reacción cruzada fueron utilizadas mezclas de sueros de BALB/c infectados con Litomosoides sigmodontis, Echinococcus multilocularis y Schistosoma mansoni a diferentes tiempos de infección: 10 semanas post infección (p.i.) para L. sigmodontis; 7 semanas p.i. para $E$. multilocularis y 8 semanas p.i. para $S$. mansoni.

Electroinmunotransferencia: La separación electroforética del antígeno en el ámbito molecular de 1,0 hasta $26,0 \mathrm{kDa}$ se realizó en una cámara vertical para electroforesis (Dual Vertical Slab Modell 220, BIORAD, Munich) según procedimiento descrito por Lueder ${ }^{9}$ con las siguientes modificaciones. Se utilizó una concentración de monómeros del $4 \%$ para el gel concentrador, del $10 \%$ para el gel espaciador y $16,5 \%$ para el gel separador. La electroforesis se efectuó con una solución amortiguadora de corrida para el cátodo (Tris $0,1 \mathrm{M}$. Tricina 0,1 M. SDS $0,1 \%$. pH 8,25) y otra para el ánodo (Tris 0,2 M. pH 8,7). Las muestras de antígeno se diluyeron 1:2 con solución amortiguadora de muestra (Tris-HCl 0,1 M. Glicerol 24\%. SDS $1 \%$. 2-Mercaptoetanol 4\%. Azul brillante de Coomasie $\mathrm{G} 0,02 \%$. pH 6,8) y hervidas por 5 minutos antes de ser separadas electroforé-ticamente. Para la identificación de los péptidos se utilizó un indicador de bajo peso molecular preteñido SIGMA. La separación electroforética se inicio con $15 \mathrm{~mA}$ por una hora y luego $30 \mathrm{~mA}$ durante 20 horas, todo bajo una temperatura de $10{ }^{\circ} \mathrm{C}$. Los patrones electroforéticos obtenidos son observados luego de utilizar la tinción de Coomassie $^{10}$.

El antígeno, separado electroforéticamente y adsorbido en el gel, es transferido a una membrana de nitrocelulosa $(0,20 \mathrm{~mm})$ en una cámara de transferencia semi-seca por 90 minutos a $0,8 \mathrm{~mA}$ por $\mathrm{cm}^{2}$ utilizando una solución 
amortiguadora de transferencia para el cátodo (Ácido 6-Aminocapronico $40 \mathrm{mM}$. Metanol 20\%. pH 7,7) y dos soluciones para el ánodo (solución A: Tris 25 mM. Metanol 20\%.pH 10,4. Solución B: Tris-cha 0,3M. Metanol 20\%.pH 10,4). Luego de la transferencia la membrana de nitrocelulosa se cortó en tiras de aproximadamente $2 \mathrm{~cm}$ de ancho bloqueándose con solución amortiguadora tris-salina (TBS: Tris $10 \mathrm{mM}$. $\mathrm{NaCl} 0,9 \%$. pH $7,4)$ con leche descremada al $5 \%$, durante toda la noche. Posteriormente las tiras se lavaron dos veces con TBS y se incubaron con los sueros en una solución de Tween-TBS/leche descremada $5 \%$, a una dilución de $1: 100$ por 2 horas a temperatura ambiente. Seguido de tres lavados con TBS y con $0,05 \%$ Tween-TBS, las tiras de nitrocelulosa con los anticuerpos adsorbidos se incubaron por 2 horas con el conjugado anti-IgA de ratón específico contra cadena $\kappa$, anti IgM específico contra cadena $\mu$, anti-IgG de ratón específico contra cadena $\gamma$ (SIGMA, Munich), anti-IgG1, IgG2a e IgG3 de ratón (Zymed, Munich), todos marcados con fosfatasa alcalina y diluidos 1: 1000 en TBS/leche descremada 5\% (excepto IgG2a e IgG3 diluidas 1:500). Las tirillas son lavadas como se mencionó anteriormente y el revelado se efectuó con el sustrato para fosfatasa alcalina: $0,33 \mathrm{mg} / \mathrm{ml} \mathrm{NBT}$ (Nitro Blue Tetrazolium) y $0,165 \mathrm{mg} / \mathrm{ml}$ BCIP (5-Bromo-4Chloro-3-Indolyl Posparte) en una solución de $\mathrm{NaCl}$ 0,1 M. $\mathrm{Mgcl}_{2}$ 5mM. Tris-HCl 0,1M. pH 9,5. La reacción se detuvo adicionando TBS.

Caracterización de los antígenos: El antígeno, separado electroforéticamente y adsorbido en el gel, se transfirió a una membrana de PVDF (polyvinyldi fluorid) en una cámara de transferencia semi-seca, por 90 minutos a 0,8 $\mathrm{mA}$ por $\mathrm{cm}^{2}$. Se utilizó la misma solución amortiguadora de transferencia para el cátodo y el ánodo: CAPS 10 mM (3-[cyclohexylamino]-1 propone-sulphonic acid), metanol al $10 \%$, tioglicolato $0,1 \mathrm{mM}, \mathrm{pH} 11,1$. Luego de la transferencia, la membrana de PVDF se lavó tres veces por diez minutos cada vez con agua bidestilada, y se tiñó con Coomassie ${ }^{10}$. Para finalizar, la membrana se lavó nuevamente con agua bidestilada según se indicó anteriormente y se dejó secar a temperatura ambiente. La membrana fue enviada al Instituto de Biología Celular, Tuebingen-Alemania, para caracterizar los antígenos de interés mediante el método de digestión de Edmann, según lo descrito por Karlson ${ }^{11}$.

\section{RESULTADOS}

La separación electroforética del extracto somático completo de A. costaricensis, mediante el Tricina-SDS-PAGE y después de teñir el gel con Coomassie, permitió observar cerca de 10 componentes proteícos con un peso molecular entre los 26,0 y 1,0 kDa. Las bandas presentes en mayor concentración, correspondieron a componentes con un peso molecular de 20 y 15 $\mathrm{kDa}$ respectivamente (Figura 1).

Los resultados indicaron que a partir de la quinta semana post-infección solamente son reconocidos por la $\operatorname{IgG}$ y $\operatorname{la} \operatorname{IgG} 1$, tres componentes con un peso molecular de 20,15 y $2 \mathrm{kDa}$ respectivamente (Figura 2). Las otras inmunoglobulinas, IgA, IgM, IgG2a e IgG3, no mostraron afinidad por los componentes antigénicos durante todo el experimento.

Para medir la reacción cruzada contra el antígeno de $A$. costaricensis fueron ensayados mezclas de sueros de animales infectados con $L$. sigmodontis, E. multilocularis y $S$. mansoni. La IgG y la IgG1 del suero de animales con infección por L. sigmodontis reconocen antígeno con un peso molecular de 15 y $2 \mathrm{kDa}$ respectivamente. Cabe destacar que la reacción observada, sobre todo para el antígeno de $15 \mathrm{kDa}$, fue muy débil y varió para cada animal. Por su parte los sueros de animales infectados con E. multilocularis y $S$. mansoni mostraron solamente una baja reactividad contra el antígeno de $2 \mathrm{kDa}$.

Para caracterizar los antígenos que mostraron mayor reactividad en la electroinmunotransferencia, se utilizó el método de digestión de Edmann. Mediante este método se obtuvo, para el componente de $2 \mathrm{kDa}$, una secuencia inicial de aminoácidos con $95 \%$ de homología con la Ubiquitina; para el componente de $15 \mathrm{kDa}$ una secuencia inicial con el $100 \%$ de homología para la enzima Glutation S-transferasa de Brugia malayi y Onchocerca volvulus; y para el componente antigénico de $20 \mathrm{kDa}$ se obtuvo una secuencia de trece aminoácidos que no correspondió a ninguna proteína hasta el momento descrita (Figura 3).

\section{DISCUSIÓN}

Una prueba de aglutinación en látex ${ }^{12}$ y una prueba inmunoenzimática (ELISA) ${ }^{5,6}$ se emplean en Costa Rica y Brasil respectivamente, para el 


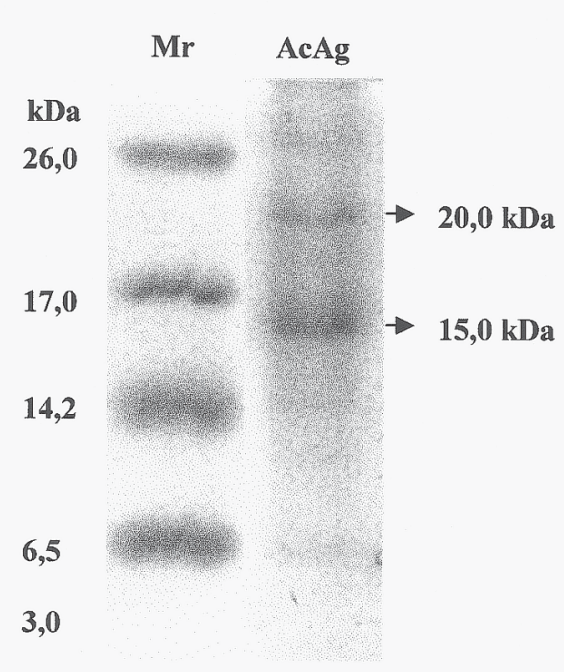

Figura 1. Separación electroforética mediante TricinaSDS-PAGE del extracto crudo de antígeno somático de Angiostrongylus costaricensis (AcAg). Se procedió a la separación analítica del AcAg en el ámbito de los 26,0 a los 1,0 kDa y a la tinción de las bandas obtenidas mediante Coomassie. Para obtener el peso molecular relativo de cada componente, se corren paralelamente marcadores (Mr) de bajo peso molecular (Ultra Low Weight Molecular Marker, 1,0-26,0 kDa, for SDS-PAGE, Sigma). A la derecha se indican los pesos moleculares de las dos bandas presentes en mayor concentración.

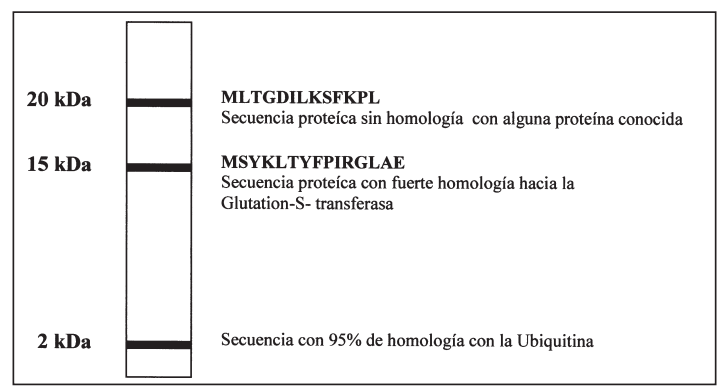

Figura 3. Diagrama que muestra la ubicación y secuencia proteíca de los tres componentes antigénicos de 2015 y $2 \mathrm{kDa}$ respectivamente, reconocidos por el suero de los animales infectados en el extracto somático crudo de $A$. costaricensis. Las secuencias proteicas fueron obtenidas mediante el método de digestión de Edmann.

diagnóstico serológico de la angiostrongilosis abdominal. Dichas pruebas utilizan como antígeno, extracto somático completo de las formas adultas de A. costaricensis. Para ambos métodos, pero especialmente para el ELISA, son frecuentes las reacciones cruzadas con otras

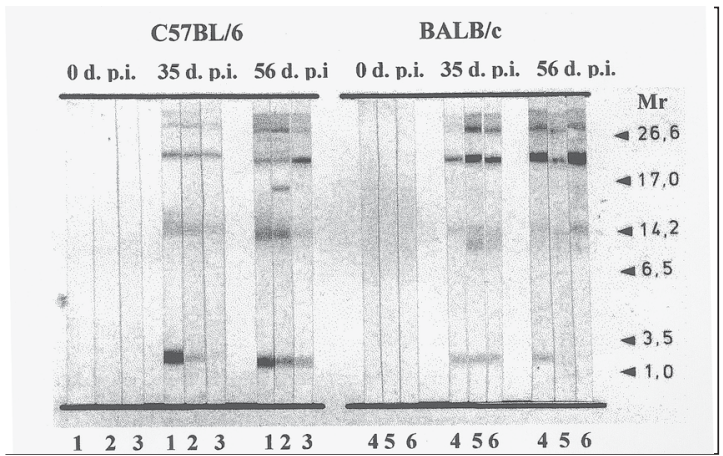

Figura 2. Proteínas de bajo peso molecular del antígeno somático de Angiostrongylus costaricensis, reconocidas por los anticuerpos IgGs de los sueros de animales infectados, C57BL/6 (1-3) y BALB/c (4-6), a los 0, 35 y 56 días post infección (d. p.i). A la derecha se indican los pesos moleculares de los marcadores (Mr) de bajo peso molecular (Ultra Low Weight Molecular Marker, 1,0-26,0 kDa, for SDS-PAGE, Sigma).

infecciones y la sensibilidad de la prueba apenas si alcanza un $85 \%{ }^{5,7}$. Otros investigadores han demostrado que gran parte de esta reacción cruzada se produce contra antígenos somáticos inmunodominates de $A$. costaricensis con un peso molecular superior a los $90 \mathrm{kDa}^{5}$. Estos autores, trabajando con sueros de pacientes infectados con el metastrongilídeo y otros parásitos (Ascaris, Trichocephalus, Strongyloides, uncinarias), determinan mediante Western Blot, una fuerte reacción cruzada contra una tripleta antigénica, en el rango de los 200-190, 140-120 y 98-93 kDa respectivamente. Resultados similares se han obtenido en el modelo animal experimental ${ }^{13}$. En este modelo se menciona el reconocimiento de antígenos inmunodominantes en el ámbito de los 80 a $210 \mathrm{kDa}$, por el suero de ratones BALB/c y C57BL/5 infectados con A. costaricensis. El autor señala además, una fuerte reacción cruzada para antígenos sobre los $85 \mathrm{kDa}$. Por el contrario, Geiger observó una respuesta humoral más específica hacia fracciones de bajo peso molecular del antígeno somático, especialmente hacia componentes bajo los $50 \mathrm{kDa}$. Con base en los resultados anteriores, se decidió llevar a cabo un estudio para identificar y caracterizar los antígenos con mayor especificidad en el ámbito del bajo peso molecular. Mediante Western blot y durante la fase crónica de la infección son reconocidas, solamente por la IgG e IgG1, tres bandas antigénicas bajo los $30 \mathrm{kDa}$ : una banda de 20 , 
otra de $15 \mathrm{y}$ una de $2 \mathrm{kDa}$ respectivamente; cabe destacar que el peso molecular de los dos primeras bandas coincide con el de los componentes presentes en mayor concentración en el extracto somático de A. costaricensis (Figura 2). Este patrón de reconocimiento antigénico fue idéntico para ambas cepas de animales y muy similar entre cada animal infectado, lo que indica la alta inmunogenicidad de estos antígenos. Por otro lado, la reacción cruzada, con excepción de la observada para la banda de $2 \mathrm{kDa}$, fue muy débil y variable, y en el caso particular de la banda de 20 kDa no se observó reacción cruzada alguna.

Mediante el método de digestión de Edmann se procedió a caracterizar cada uno de los componentes mencionados (Figura 3). Para la banda de $2 \mathrm{kDa}$ se obtuvo una secuencia que mostró un 95\% de homología con la Ubiquitina, una proteína presente en un sinnúmero de organismos, incluyendo la mayoría de los nemátodos. Es precisamente contra este componente que se observó la mayor reacción cruzada en el Western blot.

Con respecto al componente de $15 \mathrm{kDa}$, la secuencia obtenida mostró un $100 \%$ de homología con la enzima Glutation S-transferasa de las filarias B. malayi y $O$. volvulus. Lo anterior explicaría la reacción cruzada observada contra este componente la mezcla de sueros de $L$. sigmodontis, una filaria utilizada como modelo experimental de O. volvulus ${ }^{15}$, entre otras razones, por su similitud antigénica.

Es interesante que al caracterizar el componente de $20 \mathrm{kDa}$ se obtiene una secuencia de trece aminoácidos que no presenta homología con alguna proteína conocida. Esto podría indicar que se trata de una proteína específica del parásito, no descrita aún en otros helmintos.

Varios trabajos demuestran que la utilización de fracciones antigénicas, en lugar de antígeno somático completo, contribuyen a un aumento significativo de la especificidad de algunas pruebas. Por ejemplo, componentes somáticos de bajo peso molecular de Fasciola hepatica, Dirofilaria inmitis, $S$. mansoni y sobre todo de filarias, se han utilizado en el ensayo de pruebas inmunodiagnósticas que muestran una alta sensibilidad y especificidad, e incluso algunos de estos componentes han sido postulados como candidatos para el desarrollo de vacunas ${ }^{16-21}$. Los resultados del presente trabajo sugieren que, al menos en el modelo experimental, el componente de $20 \mathrm{kDa}$, además de estar presente en una alta concentración en el extracto somático de $A$. costaricensis, muestra una alta inmunogenicidad y especifidad, lo que lo convierte en un posible candidato para el desarrollo de una prueba diagnóstica. Son necesarios, sin embargo, nuevos estudios con sueros humanos para confirmar el valor diagnóstico de dicho antígeno y evaluar la factibilidad de implementarlo en una prueba de laboratorio.

\section{RESUMEN}

La respuesta inmune hacia componentes de bajo peso molecular del antígeno somático de $A$. costaricensis fue investigada en el modelo animal experimental. Durante la fase crónica de la infección y mediante el Western blot, son detectados por la IgG e IgG1 tres antígenos inmunodominantes: uno de 20 otro de 15 y uno de $2 \mathrm{kDa}$ respectivamente. Mediante el método de digestión de Edmann se obtuvo la secuencia de aminoácidos inicial de cada componente: para la banda de $15 \mathrm{kDa}$ una secuencia con un $100 \%$ de homología con la enzima glutation-Stransferasa de Brugia malayi y Onchocerca volvulus, para el componete de $2 \mathrm{kDa}$ una secuencia con $95 \%$ de homología con la proteína Ubiquitina, y para el compontente de $20 \mathrm{kDa}$ una secuencia de trece aminoácidos, que no presenta homología con alguna proteína hasta ahora descrita. En general la reacción cruzada contra estos componentes es muy débil y en el caso particular del componente de $20 \mathrm{kDa}$ no se detecta ninguna reacción cruzada. Se proponen futuros estudios con sueros humanos, para verificar la utilidad de dichos antígenos en el desarrollo de una prueba diagnóstica específica para la angiostrongiliosis abdominal.

\section{REFERENCIAS}

1.- MORERA P. Angiostrongyliasis abdominal: transmisión y observaciones sobre su posible control. In: Control and Eradication of Infections Diseases. Int.Symp. PAHO/WHO copublication 1985; Series no. 1: 230-35.

2.- MORERA P. Abdominal angiostrongyliasis: In Enteric Infection. Edt. Chapman and Hall 1995; 225-30.

3.- ORGANIZACIÓN PANAMERICANA DELA SALUD. El control de las enfermedades transmisibles en el hombre. Edt. Benenson S. A. 1987; 6-9.

4.- GRAEFF-TEIXEIRA C, CAMILLO-COURA L, LENZI H L. Clinical and epidemiological aspects of 
abdominal angiostrongyliasis in southern Brazil. Rev Inst Med Trop Sao Paulo 1991b; 33: 373-8.

5.- GRAEFF-TEIXEIRA C, AGOSTINI A A, CAMILLOCOURA L, FERREIRA-daCRUZ M F. Seroepidemiology of abdominal angiostrongyliasis: the standardization of an immunoenzymatic assay and prevalence of antibodies in two localities in Southern Brazil. Trop Med Int Hlth 1997; 2: 254-60.

6.- GEIGER S, LAITANO A C, SIEVERS-TOSTES C et al. Detection of the acute phase of abdominal angiostrongyliasis with a parasite-specific IgG enzyme linked immunsorbent assay. Mem Inst Oswaldo Cruz Rio de Janeiro 2001; 96: 515-8.

7.- GEIGER M S. Untersuchungen zur humoralen und zellulären immunantwort in der akuten phase einer experimentellen Angiostrongylus costaricensis-Infektion (Metastrongyloidea). Dissertation der Fakultät fúr Biologie der Eberhard-Karls Universität Tübingen, 1998.

8.- BRADFORD M M. A rapid and sensitive method for the quantitation of microgram quantities of protein utilizing the principle of protein-dye binding. Anal Biochem 1976; 72: 248-54.

9.- TILGNER S, METZKE H. Die Blutentnahme aus den Venen der Orbita. Zeitschr. f. Versuchstierkund 1964; 59-77.

10.- LÜDER C G K. Immunregulation bei der Onchozerkose: Untersuchungen de Onchocerca volvulus-spezifischen zellulären und humoralen Immunreaktion bei unterschiedlichen Infektions-verlaäufen. Dissertation der Fakultät für Biologie der

Eberhard-Karls-Universität Tübingen. 1995.

11.- KARLSON P. Kurzes Lehrbuch der Biochemie. 12. Auflage. Georg Thieme Verlag 1984.

12.- MORERA P. In J. A. Spittel Jr., Clinical Medice Harper \& Row, Philadelphia 1986; 3: 1-4.

13.- GEIGER M S, GRAEFF-TEIXEIRA C, SOBOSLAY T P, SCHULZ-KEY H. Experimental Angiostrongylus costaricensis infection in mice: immunoglobulin isotype responses and parasite-specific antigen recognition after primary low-dose infection. Para-sitol Res 1999; 85: 200-05.

14.- ABRAHAM S. E. Untersuchungen zur stadienspezifischen Antigenerkenung bei experimentellen
Angiostrongylus costaricensis-Infektionen (Metastrongyloidea) in Mäusen. Dissertation der Fakultät für Biologie der Eberhard-Karls-Universität Tübingen 2000.

15.- FREDES F, GORMAN T, SILVA M, ALCAINO H. Evaluación diagnóstica de fracciones cromatográficas de Fasciola hepatica mediante Western Blot y ELISA en animales infectados. Arch Med Vet 1997; 29: 283 94.

16.- HOFFMANN W, PETIT G, SCHULZ-KEY H et al. Litomosoides sigmodontis in mice: reappraisal of an old model for Filarial research. Parasitol today 2000; 16: 387-9.

17.- BLOCH P, SIMONSEN P E. Studies on immunodiagnosis of dracunculiasis. I Detection of specific serum antibodies. Acta Tropica 1998; 70: 73-86.

18.- HAMILTON J V, KLINKERT M, DOENHOFF M J. Diagnosis of schistosomiasis: antibody detection, with notes on parasitological and antigen detection methods. Parasitology 1998; S41-S57.

19.- CABRERA Z, PARKHOUSE R M E, FORSYTH K. et al. Specific detection of human antibodies to Onchocerca volvulus. Trop Med Parasitol 1989; 40; 454-9.

20.- LOBOS E, WEISS N, KARAM M et al. An immunogenic Onchocerca volvulus antigen: a specific and early marker of infection. Science 1991; 251: 603-5.

21.- HOFFMANN H W, BLANKE H C, MAIER M J et al . Antigenicity and specificity of very low molecular weight Onchocerca volvulus polypeptides in the range 2,2-12,5 kDa. Trop Med Int Htlh 1998; 2: 635-45.

Agradecimientos: Los autores desean expresar su agradecimiento al Dr. Stefan Stevanovic del Instituto de Biología Celular, Tübingen Alemania, por su valiosa colaboración para la obtención de las secuencias iniciales de aminoácidos de los antígenos de interés. Al Dr. Misael Chinchilla Carmona de la Facultad de Microbiología de la Universidad de Costa Rica por la revisión del manuscrito; y al programa fORTÜNE, Universidad de Tübingen por el soporte al estudio y al programa de servicio de intercambio académico alemán (DAAD) y Conselho para Apoio na Pesquisa (CAPES), Brasil, por el apoyo económico. 